\title{
Inferring the Fact that Static Magnetic Field Exists Along with Electrostatic Field and Conducting Experimental Verification in Accordance with the Theory of Relativity
}

\author{
Kexin Yao \& Zhisheng Wang \\ Institute of Mechanical Engineering of Shaanxi Province \\ Room 1-7-1, Staff Building, Xi'an Metering Instiution \\ No.12, Laodong South Road, Xi'an, Xi'an, 710068, P. R. China \\ Tel: 086-029-885-408-78Ｅ-mail: yayydwpq@163.com
}

\author{
Received: November 7, 2011 Accepted: November 21, 2011 Published: February 1, 2012 \\ doi:10.5539/apr.v4n1p222 \\ URL: http://dx.doi.org/10.5539/apr.v4n1p222
}

\begin{abstract}
It is considered in the superposition principle of electrostatic field and the special relativity that the electric field around the moving electrons in permanent magnet still exists and presents length contraction in the direction of movement. Through experimental analysis, it is also considered that the motion states of two electric fields having same direction and opposite direction of motion cancel out each other. Based on the two above-mentioned basic concepts, it can be inferred that negative electrostatic field exists in the vicinity of cubic permanent magnet $\mathrm{N}$ and $\mathrm{S}$ pole surface edge while positive electrostatic field exists in the vicinity of side edge. In addition, the results of experimental verification are also introduced.
\end{abstract}

Keywords: Electric field, Superposition principle, Special relativity, Permanent magnet, Self-generated electric field, Induced electric field, Analysis model

\section{Length Contraction of Electric Field in the Direction of Movement}

It is pointed out in the special relativity that when an object having the length of $\mathrm{L}$ moves at the speed of $v$, its length will be shortened as $\mathrm{L}^{\prime}=\mathrm{L} \sqrt{1-\mathrm{v}^{2} / \mathrm{C}^{2}}$ (Wherein: C- speed of light). As electric field also belongs to real matter, the length in the direction of movement will also be shortened according to the above-mentioned law when the electric field moves.

As for the cubic electric field matter locating at the latter half of electric charge Q and having side length as $\alpha$, as specified in Figure 1A. When it moves at the speed of $v$, as specified in Figure 1B, its side length in the direction of $v$ will be shortened as $\alpha \sqrt{1-\mathrm{v}^{2} / \mathrm{C}^{2}}$, and thus the volume shrinks, electric field distribution changes, field strength in the vicinity of $y$-axis increases dramatically while the field strength in the vicinity of $\mathrm{x}$-axis weakens dramatically.

Through the analysis of geometric relationship between Figure A and Figure B, and according to the principle that electric flux remains unchanged prior to and after the shrinkage of any solid angle having Q as its center, it is possible to derivate (the derivation process is omitted) that the relationship between the field strength $\mathrm{E}$ locating at $\mathrm{r}$ outside $\mathrm{Q}$ and the field strength E'locating at $\mathrm{r}$ after $\mathrm{Q}$ moves at the speed of $v$,

$$
E^{\prime}=\frac{1-v^{2} / C^{2}}{\left(1-\sin ^{2} \theta v^{2} / C^{2}\right)^{3 / 2}} E
$$

Wherein, $\theta$ is the included angle between $r$ and $v$. This equation is the same as the equation derived according to electrodynamics, which indicates that the derived analysis purely based on the geometric relationship of electric field contraction is in line with the practice result.

Equation (1) is the basic equation to be derived and analyzed in this paper. As $v$ is relatively small, $1 / \sqrt{1-v^{2} / C^{2}}=1+v^{2} / C^{2}$ (see the hereinafter explanation 3 ). Therefore, it can be known from equation (1) that the increment of field strength is $E^{\prime}-E=v^{2} / 2 C^{2}$ when $\theta=90^{\circ}$ and the reduction of field strength is 
$E^{\prime}-E=-v^{2} / C^{2}$ when $\theta=0^{\circ}$. That is, the reduction of field strength (when $v$ is relatively small and $\theta=0^{\circ}$ ) is one time higher than the increment of field strength (when $\theta=90^{\circ}$ ). It is not difficult to prove that $E^{\prime}=E$ when $v$ is relatively small and $\theta=54.7^{\circ}$. If $\theta<54.7^{\circ}, E^{\prime}<E$; if $\theta>54.7^{\circ}, E^{\prime}>E$.

\section{Macro Regular Electron Motion is Accompanied by Macroscopic Motion Electric Field}

When the combined electrical field strength of two charges of same or different polarity at a certain point are calculated in electromagnetics, it is considered that the respective electrostatic fields of these two charges are the same as that of these two charges when they exist separately. Thus, when the combined electrical field strength of two charges at a certain point is calculated, it is necessary to calculate the field strength of this point when one charge exists separately, and then calculate the field strength of this point when another charge exists separately. Finally, combine these two field strengths to serve as the total field strength of this point. This kind of combination is called the principle of superposition. Practice has proved that the principle of superposition is still correct even if the distance between these two charges is extremely small. This indicates that the existence of electric field around one charge is free from the impact of any surrounding charges. It can be inferred that all the negative electric field of electron and positive electric field of proton in all matters still exist, and the distribution of electric field is the same as that all electrons and protons exist separately. Thus, if a certain part of electrons in matter makes regular macroscopic motion, the electric fields around these electrons are bound to make the same movement along with electrons.

\section{Analysis of Interaction Force of Parallel Current-carrying Wires}

For sake of analysis and explanation, it is necessary to analyze the interaction force of simple "infinite" straight parallel current-carrying wires. In macroscopic view, a piece of lead wire can be seen as two parts. One part is negative electron beam composed of free electrons while the other part is the lead wire in which free electrons are skimmed. Electrons are bound to have positive electricity having the same quantity of electricity as negative beam. To facilitate the analysis, we call this lead wire whose free electrons are skimmed as positron beam. In this way, the current-carrying lead wires can be considered to comprise both negative beam and positron beam, and their respective electric fields are the same as that when these two exist separately. Wire current exists in the lead wire. In other word, directional movement exists in the negative beam. That is, movement is generated in the electric field around negative beam.

Figure 2 A1A2, B1B2 represent one section of two parallel A, B "infinite" straight lead wires.

In order to describe it iconically, we use pipe $\mathrm{A}+$ and $\mathrm{B}+$ in the Figure to represent the lead wires whose free electrons are skimmed, i.e, positron beam; Fluids $\mathrm{A}^{-} \mathrm{B}^{-}$in the pipeline represent the free electrons making directional movement inside the lead wire. i.e, egative beam.

To facilitate the analysis, we suppose that the kinematic velocity of all free electrons of lead wire $\mathrm{A}$ is $v_{A}$, the kinematic velocity of all free electrons of lead wire $\mathrm{B}$ is $v_{B}$. Let's use $\tau_{A}^{+}, \tau_{B}^{+}$to respectively stand for the carrying capacity of $\mathrm{A}^{+}, \mathrm{B}^{+}$unit length, and use $\tau_{A}^{-}, \tau_{B}^{-}$to respectively stand for the carrying capacity of $\mathrm{A}^{-}, \mathrm{B}^{-}$ unit length. Obviously, $\tau_{A}^{-}=-\tau_{A}^{+} ; \tau_{B}^{-}=-\tau_{B}^{+}$. If we use $\mathrm{I}_{\mathrm{A}}$ to represent the current in lead wire $\mathrm{A}$, it is obvious that $\mathrm{I}_{\mathrm{A}}=v_{A} \tau_{A}^{-}, v_{A}=\mathrm{I}_{\mathrm{A}} / \tau_{A}^{-}$; If we use $\mathrm{I}_{\mathrm{B}}$ to represent the current in lead wire $\mathrm{B}$, we can obtain $I_{B}=v_{B} \tau_{B}^{-}$, $v_{B}=I_{B} / \tau_{B}^{-}$.

Let us analyze the acting force of lead wire A against a small lead wire $d l$ on lead wire B which is away from the distance r. Positive charge $d Q_{+}=\tau_{B}^{+} d l$ and negative charge $d Q_{-}=\tau_{B}^{-} d l$ exist inside $d l$. Seem from $d Q_{+}, A^{+}$ produces electrostatic field, and its field strength is $E_{Q+}^{A+}=K 2 \tau_{A}^{+} r_{0} / r$ (wherein, $\boldsymbol{r}_{0=} \boldsymbol{r} / \boldsymbol{r}, \boldsymbol{r}_{0}$ stand for the unit vector in the direction of $\mathrm{r} ; k=1 / 4 \pi \varepsilon_{0}=9 \times 10^{9} \mathrm{Nm}^{2} / \mathrm{C}^{2} ; \varepsilon_{0}-$ permittivity of vacuum). As $A^{-}$moves at the speed of $v_{A}$, the electric field of $A^{-}$will contract as per the ratio of $\sqrt{1-v^{2} / C^{2}}$ in the direction of $v_{A}$. For this regard, the electric field density increases, and the field strength enlarges. Obviously, seen from $\mathrm{dQ}_{+}$, the field strength of $A^{-}$should be,

$$
\begin{gathered}
\boldsymbol{E}_{Q+}^{A+}=K \frac{2 \tau_{A}^{-}}{r} \frac{\boldsymbol{r}_{\boldsymbol{0}}}{\sqrt{1-v_{A}^{2} / C^{2}}} \\
=-K \frac{2 \tau_{A}^{+}}{r} \frac{\boldsymbol{r}_{\boldsymbol{\theta}}}{\sqrt{1-v_{A}^{2} / C^{2}}}
\end{gathered}
$$

Therefore, the combined field strength of $\mathrm{dQ}_{+}$should be, 


$$
\begin{array}{r}
\boldsymbol{E}_{Q+}=\boldsymbol{E}_{Q+}^{A+}+E_{Q+}^{A-} \\
=2 k \tau_{A}^{+}\left(1-\frac{1}{\sqrt{1-v^{2} / C^{2}}}\right) \frac{\boldsymbol{r}_{\boldsymbol{0}}}{r}
\end{array}
$$

Whereas, $1 / \sqrt{1-v^{2} / C^{2}}$ can be unfolded as per binomial series.

$1+v_{A}^{2} / 2 C^{2}+3 v_{A}^{4} / 8 C^{4}+5 v_{A}^{6} / 16 C^{6}+\ldots$. When $v_{A} / \mathrm{C}$ is is relatively small, $v_{A}^{4} / C^{4}, v_{A}^{6} / C^{6}$ becomes extremely small and can be omitted,thus when $v_{A}$ is relatively small,we can obtain

$$
\frac{1}{\sqrt{1-v_{A}^{2} / C^{2}}}=1+\frac{v_{A}^{2}}{2 C^{2}}
$$

Calculation shows that if $v_{A}<10^{-1} C$, the error in equation(4)is less than $3.78 \times 10^{-5}$ In other word, we can obtain the following equation if the equation(4)is substituted into equation $\mathrm{E}_{\mathrm{Q}_{+}}$:

$$
\boldsymbol{E}_{Q+}=-K \tau_{A}^{+} \frac{v_{A}^{2}}{C^{2} r} \boldsymbol{r}_{\boldsymbol{0}}
$$

The force received by $\mathrm{dQ}_{+}$should be,

$$
d \boldsymbol{F}_{Q_{+}}=E_{Q+} d Q_{+}=-K \tau_{A}^{+} \tau_{B}^{+} d l \frac{v_{A}^{2}}{C^{2} r} \boldsymbol{r}_{\boldsymbol{o}}
$$

It is indicated in equation(6)that $d \boldsymbol{F}_{Q_{+}}$is negative value,i.e. the stress direction of $\mathrm{dQ}_{+}$is opposite to the direction of $\boldsymbol{r}_{\boldsymbol{0}}$, i.e. $\mathrm{dQ}_{+}$receives the gravitational force of A current-carrying lead wire.

Let us observe the electric field force $d F_{Q_{-}}$of A received by negative charge dQ.in $d l$. As for dQ., $\mathrm{A}^{+}$moves at the speed of $-v_{B}$, and thus the field strength of $\mathrm{A}^{+}$v.s dQ. should be: $E_{Q-}^{A+}=K 2 \tau_{A}^{+} \boldsymbol{r}_{\boldsymbol{\theta}} / r \sqrt{1-v_{B}^{2} / C^{2}}$; As $\mathrm{A}^{-}$vs. dQ.moves at the speed of $v_{A}-v_{B}$, and thus the field strength of A ${ }^{-} v . s$ dQ.should be:

$E_{Q-}^{A-}=k 2 \tau_{A}^{-} \boldsymbol{r}_{\boldsymbol{0}} / r \sqrt{1-\left(v_{A}-v_{B}\right)^{2} / C^{2}}$ In the light of binomial series expansion method in the previous equation(4),we can obtain:

$$
1 / \sqrt{1-v_{B}^{2} / C^{2}}=1+v_{B}^{2} / 2 C^{2}
$$

and $1 / \sqrt{1-\left(v_{A}-v_{B}\right)^{2} / C^{2}}=1+\left(v_{A}-v_{B}\right)^{2} / 2 C^{2}$, substitute this two relational expressions into $E_{Q_{-}^{A+}}$ and $E_{Q^{-}}^{A-}$, and substitute $\tau_{A}^{-}=-\tau_{A}^{+}$and $d Q_{-}=-d Q_{+}=-\tau_{B}^{+} d l$ into the following equation, we can obtain

$$
\begin{array}{r}
\quad d \boldsymbol{F}_{Q_{-}}=\left(\boldsymbol{E}_{Q_{-}}^{A+}+\boldsymbol{E}_{Q_{-}}^{A-}\right) d Q_{-} \\
=K \frac{\tau_{A}^{+} \tau_{B}^{+}\left(v_{A}^{2}-2 v_{A} v_{B}\right) d l}{C^{2} r} \boldsymbol{r}_{\mathbf{0}}
\end{array}
$$

The total force $\mathrm{dF}$ received by $d l$ section on B current-carrying lead wire is the sum of electric field force received by $\mathrm{dQ}_{+}$and $\mathrm{dQ}_{\text {-. }}$ i.e

$$
\begin{gathered}
d F=d F_{Q_{+}}+d F_{Q_{-}} \\
=-k \frac{v_{A}^{2} \tau_{A}^{+} \tau_{B}^{+} d l}{C^{2} r} \boldsymbol{r}_{0}+k \frac{\left(v_{A}^{2}-2 v_{A} v_{A}\right) \tau_{A}^{+} \tau_{B}^{+} d l}{C^{2} r} \boldsymbol{r}_{0} \\
=-\frac{K}{C^{\mathbf{2}}} \frac{\mathbf{2} v_{A} v_{B} \tau_{A}^{+} \tau_{B}^{+} d l}{r} \boldsymbol{r}_{0}
\end{gathered}
$$


As the direction of specified current $\mathrm{I}_{\mathrm{A}}, \mathrm{I}_{\mathrm{B}}$ is opposite to the direction of $v_{A}$ and $v_{B}$, we can obtain,

$$
v_{A} \tau_{A}^{+}=-I_{A}, v_{B} \tau_{B}^{+}=-I_{B} \text {.i.e. } v_{A} v_{B} \tau_{A}^{+} \tau_{B}^{+}=I_{A} I_{B}
$$

As $k=1 / 4 \pi \varepsilon_{0} ; C^{2}=1 / \mu_{0} \varepsilon_{0}\left(\mu_{0}\right.$-space permeability $)$,and thus:

$$
\frac{k}{C^{2}}=\frac{1}{4 \pi \varepsilon_{0}} \mu_{0} \varepsilon_{0}=\frac{\mu_{0}}{4 \pi}
$$

Substitute the above-mentioned relational expression into the equation(8), we can obtain,

$$
d \boldsymbol{F}=-\frac{\mu_{0}}{4 \pi} \frac{2 I_{A} I_{B} d l}{r} \boldsymbol{r}_{0}
$$

The force $F_{l}$ received by the lead wire(its length is L) on B current-carrying lead wire should be:

$$
\boldsymbol{F}_{l}=\int_{0}^{l} d F=-\frac{\mu_{0}}{4 \pi} \frac{2 I_{A} I_{B} l}{r} \boldsymbol{r}_{\boldsymbol{0}}
$$

The negative sign on the right end of equal sign in equation (11) represents that $\boldsymbol{F}_{l}$ is negative value, and its direction is opposite to the direction of $\boldsymbol{r}_{\boldsymbol{o}}$ if $\mathrm{I}_{\mathrm{A}}$ and $\mathrm{I}_{\mathrm{B}}$ share the same direction; $\boldsymbol{F}_{l}$ is positive value, its direction is the same as the direction of $\boldsymbol{r}_{\boldsymbol{0}}$, and $\mathrm{B}$ will be rejected by $\mathrm{A}$ if $\mathrm{I}_{\mathrm{A}}$ and $\mathrm{I}_{\mathrm{B}}$ are negative values [the previous $\left(v_{A}-v_{B}\right)$ becomes into $\left(v_{A}+v_{B}\right), \mathrm{dF}$ in equation (8)turns from negative into positive]. It is obvious that this equation(11)is identical to the electromagnetic inference.

The above $v_{A}$ and $v_{B}$ are the average kinematic velocity of free electrons in A and B lead wires. If the analysis is based on the actual free electron movement,the equation(11)still holds water. It is briefly proved as follows,

Suppose $\mathrm{I}_{\mathrm{A}}$ comprises $m$ group small current beam having identical speed of free electron

$\Delta \mathrm{I}_{\mathrm{A} 1}, \Delta \mathrm{I}_{\mathrm{A} 2} \ldots \Delta \mathrm{I}_{\mathrm{Am}}$ composition,

$I_{A}=\sum_{i=1}^{m} \Delta I_{A i} ;$ Similarly, $I_{B}=\sum_{i=1}^{n} \Delta I_{B j}$

The mutual acting force between any two small current beams $\Delta \mathrm{I}_{\mathrm{Ai}}$ and $\Delta \mathrm{I}_{\mathrm{Bj}}$ complies with the equation(11), and thus the mutual acting force between each of small current beams in all $I_{A}$ and $I_{B}$ also complies with the equation(11).

$\boldsymbol{F}_{L}=-\frac{\mu_{0} 2 l}{4 \pi r} \boldsymbol{r}_{0} \sum_{i=1}^{m} \sum_{j=1}^{n} \Delta I_{A i} \Delta I_{B i}$ is sure to come into existence, and $\sum_{i=1}^{m} \sum_{j=1}^{n} \Delta I_{A i} \Delta I_{B j}=I_{A} I_{B}$

i.e the equation(11) still comes into existence. That is to say,the uneven distribution of free electron velocity has no impact on the correctness of conclusion in the equation(11).

\section{The Motion State of Two Electric Fields Having the Same Direction and Opposite Direction of Motion Cancels out Each Other}

The interaction force of two parallel current-carrying lead wires is inferred and deduced in the above context. We can also deduce the Ampere force law under the conditions of uniform magnetic field in accordance with the view of electric field contraction (it is not to be introduced here due to relatively burdensome deduction process). Thus, under normal circumstances, the view of electric field contraction is not contradictory to the electromagnetics. However, we can also see the special deduction of electric field contraction. Refer to the previous equation (5), which indicates that the electric field (generates from the current-carrying lead wire) perceived by the static charge $\mathrm{dQ}_{++}$is $E_{Q_{+}}=-k \tau_{A}^{+} v_{A}^{2} r_{0} / c^{2} r$. Can this electric field be detected through an experiment? The calculation shows that the electric field is extremely weak, in other word, it can not be measured. For example, when the copper lead wire having sectional area of $100 \mathrm{~mm}^{2}$ is charged with extremely large $1000 \mathrm{~A}$ current, it is possible to calculate that the field strength of the site $10^{-2} \mathrm{~m}$ away from this lead wire is only $7.387 \times 10^{-6} \mathrm{v} / \mathrm{m}$. In other word, it is equivalent to the field strength between two plates when two "infinite" metal plates $200 \mathrm{~km}$ away with each other are connected with a $1.5 \mathrm{~V}$ battery. Such small field strength is certainly very difficult to be determined. (The reason why another lead wire B receives obvious stress lies in the fact that the quantity of electricity of proton and electron in B is huge). 
It can be found in the above analysis of $\boldsymbol{E}_{Q_{+}}$that we can obtain the following equation if $v_{A} \tau_{A}^{+}=I_{A}$ is substituted into $\boldsymbol{E}_{Q+}=-K v_{A} I_{A} \boldsymbol{r}_{\boldsymbol{o}} / c^{2} \boldsymbol{r}$. It can be known that $\mathrm{E}_{\mathrm{Q}+}$ can be detected if $v_{A}$ is very huge under the condition that current $\mathrm{I}_{\mathrm{A}}$ remains unchanged. It is obviously not much too huge in the lead wire $v_{A}$. However, in the magnetic domain of permanent magnet, the spin speed of electron is extremely huge. Normally, its speed is $10^{6} \mathrm{~m} / \mathrm{s}$. This huge speed naturally owns relatively stronger $\boldsymbol{E}_{Q_{+}}$.

The author applies a cubic neodymium iron boron permanent magnet (surface magnetic induction is about .08T, side length is $20 \mathrm{~mm}$ ) as test sample. The calculation shows that the magnetic field generated by this test sample is the same as that generated by one turn of square coil (field strength is 29209A), we can thus use a turn of square coil (formed by a square face having even distribution of surface current, and $20 \mathrm{~mm}$ side length on each face) as an analysis model.

In the light of the method applied in the analysis of current-carrying lead wire motional electric field, we use each face in the above model as a section of lead wire in the analysis. From the analysis, we can see that the field density component of motional electric field generated by negative electron motion (current) in NS axis direction in four faces of analysis model is the same, and its size of field strength is correlated to the average speed of spinning electron. It is known that the spinning speed of electron is $10^{6} \mathrm{~m} / \mathrm{s}$, and the spin condition of all electrons of permanent magnet is far from being exactly the same. Therefore, its average speed should be far less than $10^{6} \mathrm{~m} / \mathrm{s}$. The author supposes that the average speed is between $10^{3}$ and $10^{5} \mathrm{~m} / \mathrm{s}$, and calculates that the combined field strength at $10 \mathrm{~mm}$ above $\mathrm{N}$ or S face as per 29209A (the detailed calculation is omitted, and the calculation method can be referred in 5) is $-2.26 \times 10^{2} \sim-2.26 \times 10^{4} \mathrm{v} / \mathrm{m}$; The combined field strength at $5 \mathrm{~mm}$ above $\mathrm{N}$ or $\mathrm{S}$ face is $-2.99 \times 10^{2} \sim 2.99 \times 10^{4} \mathrm{v} / \mathrm{m}$. This field strength can be easily detected (detection method is shown in 6).

However, it is surprising that the conclusion of determination, after hundreds of experiments, can only be the result that electric field does not exist at the center outside $\mathrm{N}$ or S plane, and it is extremely small even if it exists. The author is puzzled on this experiment, and spends more than two years in plunging into the state of being perplexed, hesitated, and at a loss. He found the direction to solve the problem only after he realized that the moving electric field is the magnetic field. It is pointed out in the Biot-Savart Law that,

$$
d \boldsymbol{B}=-\frac{\mu_{0}}{4 \pi} \frac{I d \boldsymbol{l} \times \boldsymbol{r}_{0}}{r^{2}}
$$

Actually, the current I in lead wire is formed by the average speed of $-\boldsymbol{v}$ of free electron in lead wire moving at the direction opposite to I. If we use $-\tau$ to represent the linear electric charge of free electron,we can obtain $I d l=-\tau d l(-v)=d Q_{+} v$. In the previous equation(8), we have $\mu_{0} / 4 \pi=K / C^{2}$, so, we substitute these relational expressions into dBand obtain the following,

$$
d \boldsymbol{B}=\frac{K}{C^{2}} \boldsymbol{v} \times \frac{d Q}{r^{2}} \boldsymbol{r}_{\boldsymbol{o}}=\frac{1}{C^{2}} \boldsymbol{v} \times d \boldsymbol{E}
$$

the above equation can also be written as:

$$
\boldsymbol{B}=\frac{1}{C^{2}} \boldsymbol{U} \times \boldsymbol{E}
$$

It is indicated in this equation that the moving electric field is the magnetic field (protons in the lead wire does not move, and has nothing to do with the electric field). The experiment shows that the magnetic field does not exist around a section of folded current-carrying lead wire. In other word, the moving electric field doe not exist around this current-carrying lead wire. However, two macro-free-electron groups having opposite direction of motion do exist in this lead wire. If the surrounding of free-electron groups is accompanied by free electron movement, the moving electric field is bound to exist. However, the experiment proves that the magnetic field does not exist, in other word, the moving electric field does not exist either. It means the motion state of two electric fields having the same direction and opposite direction of motion cancels out each other. Therefore, we draw the conclusion that the motion state of two electric fields having the same direction and opposite direction of motion cancels out each other. From this conclusion, we can easily understand the reason why the electric field can not be detected at the center outside $\mathrm{N}$ or $\mathrm{S}$.

It is explained in the previous context that one turn of coil (electrons uniformly distribute on the surface) can be used as an analysis model to test the permanent model. Four current surfaces of this analysis model can be respectively represented by A, B, C, D. Let us observe the electric field combination between face A and face C. Obviously, the field strength component of moving electric field (generated by electron movement) in $\mathrm{N}$ and $\mathrm{S}$ central axis is the same in size and opposite in direction. It is inferred according to the previous cancellation that 
the motion state of moving electric field is mutually cancelled with each other. Therefore, the combined negative electric field of these two belongs to electrostatic field, and the combined field strength of positive and negative electric field is zero. For the same reason, the combined field strengths of positive and negative electric field on face B and face D are also zero. It can be seen that four faces of A, B,C and D, in other word, the combined field strengths of whole coil on $\mathrm{N}$ and $\mathrm{S}$ central axis are zero. So, it is naturally that the existence of electric field can not be detected in the experiment. It is obvious that the moving electric fields can not be completely cancelled in other spaces which are not on $\mathrm{N}$ and $\mathrm{S}$ central axis. Relatively larger combined field strength may exist in some space.

\section{Calculation of Combined Field Strength at Several Basic Positions Around Rectangle Coil}

\subsection{Combined field strength along the N.S direction outside rectangle coil}

5.1.1 Calculation of positive, negative field strength along the N.S direction outside coil

A, B, C, and D in Figure 3 represent current surface analysis model of the square with side length being $2 \alpha$. The four current surfaces constitute a rectangle coil with only one turn. When the coil current is 29209A, the field strength is the same as that of a permanent magnet of the same shape with the surface magnetic induction intensity of $0.8 \mathrm{~T}$. The current direction of the coil specified in the figure is vertical with $\mathrm{y}$, and the direction of magnetic field at the center of the coil is in parallel with $\mathrm{y}$. y goes through the central line mn of A surface. $\mathrm{h}$ is used to represent $\mathrm{O}_{1} \mathrm{~m}$, and we take $\mathrm{h}_{1}=5 \times 10^{-3} \mathrm{~m}$ and $\mathrm{h}_{2}=5 \times 10^{-2} \mathrm{~m}$ in the experiment; $\mathrm{D}$ is sued to represent $\mathrm{O}_{1} \mathrm{p}$, and we take $\mathrm{D}=2 \times 10^{-3} \mathrm{~m}$ in the experiment. Now we need to calculate out the combined field strengths of electron and proton on the four surfaces $\mathrm{A}, \mathrm{B}, \mathrm{C}$, and $\mathrm{D}$ of the coil on the $\mathrm{P}_{1}$ point along the direction of $\mathrm{y}$.

Let's look at the surfaces of $\mathrm{B}$ and $\mathrm{D}$ first. Since $\mathrm{P}_{1}$ point is located in the middle of $\mathrm{B}$ and $\mathrm{D}$ surfaces, the values and directions of the field strengths of motion electric fields generated from electron motion on B surface and D surface on the $\mathrm{P}_{1}$ point along the direction of $\mathrm{y}$ are the same, and their motion directions are opposite to each other (their current directions are opposite). Because their motions are offset mutually, their negative electric field is equal to static negative field. Therefore, we can know that the combined field strength of the negative and positive electric fields (generated by the protons of the same electric quantity with motion electron) of B surface and $\mathrm{D}$ surface on the $\mathrm{P}_{1}$ point along the direction of $\mathrm{y}$ is zero. Thus, we do not need to consider the combined field strength of B surface and D surface, and only need to analyze that of A surface and C surface.

$\sigma_{+}$and $\sigma_{-}$represent the charge surface density of each motion electron of the coil and that the proton of the same electric quantity with the motion electron. It is obvious that $\sigma_{-}=--\sigma_{+}, v$ represents the motion speed of the electron, and $\sigma_{-}=-I / 2 a v$. Let's first analyze the field strength of surface A and the proton of the same electric quantity with the motion electron on the $\mathrm{P}_{1}$ point along the direction of $\mathrm{y}$.

As specified in the figure, the electric quantity of micro-surface current element $d x d y$ on $\mathrm{j}$ point and the proton of the same electric quantity with the motion electron is $d Q_{+}=\sigma_{+} d x d y$. The field strength of $d Q_{+}$on the $\mathrm{P}_{1}$ point along the direction of $y$ is:

$$
d E_{A 1}^{+}=K \frac{d Q}{r^{2}} \frac{y}{r}=\frac{k \sigma_{+} y d x d y}{r^{3}}
$$

We can know from the figure: $r=\sqrt{x^{2}+y^{2}+D^{2}}$

$$
\begin{gathered}
E_{A 1}^{+}=\iint d E_{A 1}^{+}=K \sigma_{+} \int_{h}^{h+2 a} y a y \int_{-a}^{+a} \frac{d x}{\left(x^{2}+y^{2}+D^{2}\right)^{3 / 2}} \\
=2 K \sigma_{+} \int_{h}^{h+2 a} \frac{a y d y}{\left(D^{2}+y^{2}\right) \sqrt{D^{2}+y^{2}+a^{2}}} \\
=2 K \sigma_{+}\left[\ln \frac{\sqrt{D^{2}+y^{2}}}{a+\sqrt{D^{2}+y^{2}+a^{2}}}\right]_{h}^{h+2 a}
\end{gathered}
$$

As for $\mathrm{P}_{1}$ piont, $\mathrm{D}$ of surface A will increase to $\mathrm{D}+2 \mathrm{a}$ on surface $\mathrm{C}$. Equation (13) is still applicable to the calculation of $E_{C}^{+}$of surface $\mathrm{C}$, and only $\mathrm{D}$ is changed into D+2a. 
Let's analyze the motion electric field strength of the electron motion of surface $A$ and $C$ the $\mathrm{P}_{1}$ point along the direction of $\mathrm{y}$. First look at the field strength of $E_{A 1}^{-}$on surface A. The front $\mathbf{1}$ indicates that the relationship between the field strength $\mathrm{E}$ locating at $\mathrm{r}$ outside $\mathrm{Q}$ and the field strength $\mathrm{E}$ ' locating at $\mathrm{r}$ after $\mathrm{Q}$ moves at the speed of $v$ is equation (1): $E^{\prime}=E\left(1-v^{2} / C^{2}\right) /\left(1-\sin ^{2} \theta v^{2} / C^{2}\right)^{3 / 2}$. Therefore, $d E_{A 1}^{-}$of the field strength of electron motion on $\mathrm{x}$ and $\mathrm{y}$ points of Figure 3 should be:

$$
d E_{A 1}^{-}=\frac{k \sigma_{-} y d x d y}{r^{3}} \frac{\left(1-v^{2} / C^{2}\right)}{\left(1-\sin ^{2} \theta v^{2} / C^{2}\right)^{3 / 2}}
$$

Wherein, $\theta$ is the included angle between $\mathrm{r}$ and $v$. We can know from the figure: $\sin \theta=\sqrt{D^{2}+y^{2}} / r$ is substituted into $d E_{A 1}^{-}$, and we can assume $n=\sqrt{1-v^{2} / c^{2}}$. The equation is simplified into:

$$
\begin{gathered}
d E_{A 1}^{-}=\frac{k \sigma_{-} n^{2} y d y d x}{\left[x^{2}+\left(D^{2}+y^{2}\right) n^{2}\right]^{3 / 2}} \\
E_{A 1}^{-}=\iint d E_{A 1}^{-}=2 K \sigma_{-}\left[\ln \frac{\sqrt{D^{2}+y^{2}}}{\frac{a}{n}+\sqrt{\left(D^{2}+y^{2}\right)+\frac{a^{2}}{n^{2}}}}\right]_{n}^{n+2 a}
\end{gathered}
$$

$E_{C 1}^{-}$relationship expression is similar to $E_{A 1}^{-}(14)$, and only $D^{2}$ in (14)is changed into $(D+2 \alpha)^{2}$.

5.1.2 Calculation of combined field strength

Surface A and surface B should be analyzed as a whole. Current directions on surface A and surface B are opposite, and the directions of motion electric fields generated by electron motion on the $\mathrm{P}_{1}$ point along the direction of y are opposite of course. But the directions of their electric fields are the same, so they are offset mutually. Their combined field strength should be $E_{A}^{-}-E_{C}^{-}$. If there is no offsetting effect, their combined field strength should be $E_{A}^{-}+E_{C}^{-}$. This means that through the offsetting, among the motion electrons on surface $\mathrm{A}$ and surface $\mathrm{B}$, only $\left(E_{A}^{-}-E_{C}^{-}\right) /\left(E_{A}^{-}+E_{C}^{-}\right)$generates motion electric field on the $\mathrm{P}_{1}$ point along the direction of $\mathrm{y}$. The field strength generated by the proton of the same electric quantity with the electron on the $\mathrm{P}_{1}$ point along the direction of y should be:

$$
\left(E_{A}^{+}+E_{C}^{+}\right) \times\left(E_{A}^{-}-E_{C}^{-}\right) /\left(E_{A}^{-}+E_{C}^{-}\right)
$$

We can know from this that the combined field strength of the electron and proton of surface A and surface $\mathrm{C}$ on the $\mathrm{P}_{1}$ point along the direction of $\mathrm{y}$ should be:

$$
E_{A C}=\left(E_{A}^{-}-E_{C}^{-}\right)\left(1+\frac{E_{A}^{+}+E_{C}^{+}}{E_{A}^{-}+E_{C}^{-}}\right)
$$

[Note: $\left(E_{A}^{+}+E_{C}^{+}\right) /\left(E_{A}^{-}+E_{C}^{-}\right)$is a negative value]

To make it corresponding to the experimental results and easy for distinction, when the following $\mathrm{h}=5 \times 10^{-3 \mathrm{~m}}$, $\mathcal{E}_{1}$ represents $\mathrm{E}_{\mathrm{AC}}$; when $\mathrm{h}=10^{-2 \mathrm{~m}}, \quad \mathcal{E}_{2}$ represents $\mathrm{E}_{\mathrm{AC}}$.

\subsection{Combined field strength vertical with the $N \cdot S$ direction outside the coil}

Figure 4 indicates the current direction of the coil is in parallel with y; the direction of the central magnetic field is vertical with y. This is equivalent to the coil in Figure 3 rotating $90^{\circ}$. We need to calculate out the combined field strength of $\mathrm{P}_{3}$ point along the direction of $\mathrm{y}$.

Since surfaces A, B, C, and D are square, the field strengths $E_{A 3}^{+}, E_{C 3}^{+}, E_{A 4}^{+}$, and $E_{C 4}^{+}$generated by the proton of surface $\mathrm{A}$ and surface $\mathrm{B}$ on the $\mathrm{P}_{3}$ point along the direction of $\mathrm{y}$ are the same as those in Figure 3, namely $E_{A 3}^{+}=$ $E_{A 1}^{+} ; E_{C 3}^{+}=E_{C 1}^{+} ; E_{A 4}^{+}=E_{A 2}^{+}$; and $E_{C 4}^{+}=E_{C 2}^{+}$.

Let's look surfaces B and D. It is obvious that there is no completely offsetting effect on surfaces B and D for $\mathrm{P}_{3}$. So we have to consider the combined field strength of surface B and D. Comparison shows that $h$ of surface B is 
equivalent to $D$ of surface $A$; D of surface $B$ is equivalent to $h$ of surface $A ; Z$ of surface $B$ is equivalent to y of surface $A$. The fundamental difference is: the component of $d E$ on surface $B$ along the direction of $y$ is $h / r$, and $h$ is a constant. Hence, for surface B, the field strength of the surface's micro-current surface positive charge $d Q_{+}=\sigma_{+} d x d z$ on the $\mathrm{P}_{3}$ point along the direction of $\mathrm{y}$ is:

$$
d E_{B 3}^{+}=k \frac{d Q_{+}}{r^{3}} \frac{h}{r}
$$

We can know from the figure: $r=\sqrt{x^{2}+z^{2}+h^{2}}$, substitute $\mathrm{r}$ and $\mathrm{dQ}_{+}$into $d E_{B 3}^{+}$, and obtain after integration:

$$
E_{B 3}^{+}=\iint d E_{B 3}^{+}=\int_{D}^{D+2 a} d z \int_{-a}^{+a} \frac{h d x}{\left(x^{2}+z^{2}+h^{2}\right)^{3 / 2}}=2 K \sigma_{+}\left[\sin ^{-1}\left(\sqrt{\frac{a^{2}}{a^{2}+h^{2}}} \sin \operatorname{tg}^{-1} \frac{z}{h}\right)\right]_{D}^{D+2 a}
$$

The difference between surface D and surface B is: h of surface B is $2 \alpha+h$ on surface D. Therefore, $h$ in equation (19) is changed into $2 \alpha+\mathrm{h}$, and we can calculate out $E_{D 3}^{+}$.

When $\mathrm{E}_{\mathrm{B} 3}^{+}$and $\mathrm{E}_{\mathrm{D} 3}^{+}$represent $\mathrm{h}=5 \times 10^{-3 \mathrm{~m}}$, the field strength is represented with $E_{B 4}^{+}$and $E_{D 4}^{+}$separately when $\mathrm{h}=10^{-2} \mathrm{~m}$.

Let's now calculate out motion field strengths generated by electron motions of surfaces A, B, C, and D on the $\mathrm{P}_{3}$ point along the direction of $\mathrm{y}$. Figure 4 shows that since I of surface $\mathrm{A}$ is in parallel with $\mathrm{y}$, the included angle $\theta$ between $\mathrm{r}$ and $\mathrm{I}$ is becoming smaller obviously. The above 1 points out that if $\theta<54.7^{\circ}, \mathrm{E}^{\prime}<\mathrm{E}$. This means $\left|E^{-}\right|<$ $\left|E^{+}\right|$, namely $E_{A}^{-}+E_{A}^{+}>0$, which indicates that the combined field strength of surface $\mathrm{A}$ on $\mathrm{P}_{3}$ point is a positive value. After the analysis we know that among the combined field strength of surfaces $\mathrm{A}, \mathrm{B}, \mathrm{C}$, and $\mathrm{D}$ on the $\mathrm{P}_{3}$ point along the direction of $\mathrm{y}$, the effect of surface $\mathrm{A}$ is dominant. Therefore, the total combined field strength of the four surfaces might be a positive value.

Let's analyze surface first. Through the comparison with Figure 4 and 3 we can know they are different in the fact that $\sin \theta=\sqrt{D^{2}+y^{2}} / r$ in Figure 3 and $\sin \theta=\sqrt{D^{2}+x^{2}} / r$ in figure 4. Therefore, we can obtain with $\sin \theta=\sqrt{D^{2}+x^{2}} / r$ according to equation (14): (note: $n=\sqrt{1-v^{2} / c^{2}}$ ):

$$
E_{A 3}^{-}=2 K \sigma_{-} n\left[\ln \frac{\sqrt{D^{2} n^{2}+y^{2}}}{a n+\sqrt{y^{2}+n^{2}\left(a^{2}+D^{2}\right)}}\right]_{h}^{h+2 a}
$$

Figure 4 reveals that $\mathrm{D}$ of surface $\mathrm{A}$ is $\mathrm{D}+2 \mathrm{a}$ on surface $\mathrm{C}$. Therefore, we can obtain $E_{C 3}^{-}$through changing $\mathrm{D}$ in equation (17) into D+2a. Current directions of surface $\mathrm{C}$ and $\mathrm{A}$ are opposite, and their motion electric fields have the offsetting effect. Therefore, the combined field strength of surface $\mathrm{A}$ and $\mathrm{C}$ should be calculated according to the equation 15. When $\mathrm{h}=5 \times 10^{-3 \mathrm{~m}}, \mathrm{E}_{\mathrm{AC}}$ is represented with $\varepsilon^{3}{ }_{\mathrm{AC}}$; when $\mathrm{h}=10^{-2 \mathrm{~m}}, \mathrm{E}_{\mathrm{AC}}$ is represented with $\varepsilon^{4}{ }_{\mathrm{AC}}$. Then let's look at surface B. It is mentioned above that $\mathrm{h}$ of surface $\mathrm{B}$ is equivalent to $\mathrm{D}$ of surface $\mathrm{A}$. $\sin \theta=\sqrt{D^{2}+x^{2}} / r$ on surface $\mathrm{A}$, so that on surface $\mathrm{B}$ :

$$
\begin{gathered}
\sin \theta=\sqrt{h^{2}+x^{2}} / r \\
d E_{B 3}^{-}=k \frac{\sigma_{-} d z d x}{r^{2}} \frac{h}{r} \frac{n^{2}}{\left(1-\sin ^{2} \theta v^{2} / C^{2}\right)^{3 / 2}}
\end{gathered}
$$

Substitute $\sin \theta=\sqrt{h^{2}+x^{2}} / r$ into the above equation, and obtain after integration:

$$
\begin{gathered}
E_{B 3}^{-}=\iint d E_{B 3}^{-} \\
=2 K \sigma_{-}\left[\sin ^{-1}\left(\sqrt{\frac{a^{2}}{a^{2}+h^{2}}} \sin \operatorname{tg}^{-1} \frac{z}{h n}\right)\right]_{D}^{D+2 a}
\end{gathered}
$$

It is obvious that we can obtain $E_{D 3}^{-}$after changing $\mathrm{h}$ into $\mathrm{h}+2 \alpha$ and substituting it into equation (21). $E_{D 3}^{-}$and $E_{B 3}^{-}$have the offsetting effect. Therefore, the combined field strength of surface $\mathrm{B}$ and $\mathrm{D}$ should also be calculated according to the equation 15 . When $\mathrm{h}=5 \times 10^{-3 \mathrm{~m}}, \mathrm{E}_{\mathrm{BD}}$ is represented with $\varepsilon_{\mathrm{BD}}$; when $\mathrm{h}=10^{-2 \mathrm{~m}}, \mathrm{E}_{\mathrm{BD}}$ is 
represented with $\varepsilon^{4}{ }_{\mathrm{BD}}$. As the current direction of surface B and D is vertical to the current direction of surface $\mathrm{A}$ and $\mathrm{C}, \mathcal{E}^{3}{ }_{\mathrm{AC}} \quad \mathcal{E}^{4}{ }_{\mathrm{AC}}$ and $\mathcal{E}^{3}{ }_{\mathrm{BD}} \mathcal{E}^{4}{ }_{\mathrm{BD}}$ have no offsetting effect, thus the total combined field strength is the sum of them, namely,

$$
\begin{aligned}
& \mathcal{E}_{3}=\mathcal{E}^{3}{ }_{\mathrm{AC}}+\boldsymbol{E}^{3}{ }_{\mathrm{BD}} \\
& \mathcal{E}_{4}=\boldsymbol{E}^{4}{ }_{\mathrm{AC}}+\boldsymbol{E}^{4}{ }_{\mathrm{BD}}
\end{aligned}
$$

\subsection{Combined field strength vertical with the central direction of the side of the coil}

Figure 5 indicates that the direction of the magnetic field at the center of the coil is in parallel with $\mathrm{y}$, the distance between $\mathrm{P}_{5}$ point and the side of the coil is $h$, and coordinate origin is at the center of surface A. Now we have to calculate out the combined field strength on the $\mathrm{P}_{5}$ point along the direction of $\mathrm{x}$.

As specified in the figure, surfaces $\mathrm{B}$ and $\mathrm{D}$ are symmetric to $\mathrm{P}_{5}$ point, and their current directions are opposite. So we can know that motion electric fields generated by motion electrons of surface $\mathrm{B}$ and surface $\mathrm{D}$ on the $\mathrm{P}_{5}$ point along the direction of $\mathrm{x}$ are the same in the value and direction and opposite in motion direction. There motion electric fields are offset completely, thus equal to a static negative electric field. Therefore, the combined field strength of electrons and protons on surface $\mathrm{B}$ and surface $\mathrm{D}$ on the $\mathrm{P}_{5}$ point along the direction of $\mathrm{x}$ is zero, which has no need to be considered. Therefore, we only analyze the combined field strength of surface A and surface $\mathrm{C}$ on the $\mathrm{P}_{5}$ point.

Let's look at surface A first. In Figure 5, the field strength of micro-current surface positive charge $d Q_{+}=\sigma_{+} d y d z \quad 5$ on the on the $\mathrm{P}_{5}$ point along the direction of $\mathrm{x}$ is:

$$
\begin{aligned}
& d E_{A 5}^{+}=k \frac{d Q_{+}}{r^{2}} \frac{h}{r} \\
& r=\sqrt{x^{2}+z^{2}+h^{2}}
\end{aligned}
$$

It is known from this that $\mathrm{r}$ and $d E_{A 5}^{+}$are similar to calculation conditions for the above equation (19), and the only difference is the limit of integration. So we can obtain:

$$
E_{A 5}^{+}=2 K \sigma_{+}\left[\sin ^{-1}\left(\sqrt{\frac{a^{2}}{a^{2}+h^{2}}} \sin \operatorname{tg}^{-1} \frac{z}{h}\right)\right]_{-a}^{+a}
$$

$\mathrm{h}$ of surface $\mathrm{A}$ is changed into $2 \alpha+\mathrm{h}$ on surface $\mathrm{C}$, and equation (21) is still applicable to $\mathrm{E}_{\mathrm{C} 5}{ }^{+}$of surface $\mathrm{C}$.

Figure 5 reveals that $\sin \theta=\sqrt{y^{2}+h^{2}} / r$, which is the same as the calculation of the above equation (21), and the only difference is the limit of integration. Compared with equation (21), we can obtain:

$$
E_{A 5}^{-}=2 K \sigma_{-}\left[\sin ^{-1}\left(\sqrt{\frac{a^{2}}{a^{2}+h^{2}}} \sin t^{-1} \frac{z}{h n}\right)\right]_{-a}^{+a}
$$

$\mathrm{h}$ of surface $\mathrm{A}$ is changed to $2 \mathrm{a}+\mathrm{h}$ in surface $\mathrm{C}$, equation (22) is still applicable to $\mathrm{E}_{\mathrm{C5}}^{-}$of surface $\mathrm{C}$. To correspond with experiments 5 and 6 , when $\mathrm{h}=5 \times 10^{-3 \mathrm{~m}}$, the corresponding field strength of surface $\mathrm{A}$ and $\mathrm{C}$ is $\mathrm{E}_{\mathrm{A} 5}^{+} \mathrm{E}_{\mathrm{A} 5}^{-}$and $\mathrm{E}_{\mathrm{C} 5}^{+} \mathrm{E}_{\mathrm{C} 5}^{-}$respectively. When $\mathrm{h}=10^{-2 \mathrm{~m}}$, the corresponding field strength of surface $\mathrm{A}$ and $\mathrm{C}$ is $\mathrm{E}_{\mathrm{A} 6}^{+}$ $\mathrm{E}_{\mathrm{A} 6}^{-}$and $\mathrm{E}_{\mathrm{C} 6}^{+} \mathrm{E}_{\mathrm{C} 6}^{-}$respectively. According to equation (15), there is:

$$
\begin{aligned}
& \varepsilon_{5}=\left(E_{A 5}^{-}-E_{C 5}^{-}\right)\left(1+\frac{E_{A 5}^{+}+E_{C 5}^{+}}{E_{A 5}^{-}+E_{C 5}^{-}}\right) \\
& \varepsilon_{6}=\left(E_{A 6}^{-}-E_{C 6}^{-}\right)\left(1+\frac{E_{A 6}^{+}+E_{C 6}^{+}}{E_{A 6}^{-}+E_{C 6}^{-}}\right)
\end{aligned}
$$

\section{Experiment Method and Result}

\subsection{Experiment method}

Figure 6 is the sketch map for test method. A is a square cube NdFeB permanent magnet with the side length 
being $20 \mathrm{~mm}$, and its surface magnetic strength is $0.8 \mathrm{~T}$; B is identical with $\mathrm{A}$ in size and material. Without magnetized, however, B is not a permanent magnet. D is a induced copper tray, and its role is to receive static induction of the electrified body so as to make the bottom $\mathrm{P}$ of the tin foil strip under it induce the charge of the same nature with the electrified body. Through adjustment, D can move leftward, rightward, forward, and backward, and tin foil strip can move upward and downward so as to position P anywhere between A and B. L and $\mathrm{R}$ are flat metal plates with large areas, and able to generate even electric field in the space between them after they are connected with DC power. The direction of electric field between $\mathrm{L}$ and $\mathrm{R}$ will change with the change of switch direction. Figure 7 is the photo of the experiment devices.

As specified in Figure 6, when $\mathrm{P}$ is charged, the surface of A close to $\mathrm{P}$ will have electric charge, and further generate electric field, which is defined as the induced electric field. The role of B is to generate an induced electric field of the same size but different direction with the induced electric field of A so as to make them offset.

According to the calculation method of 5 , when $\mathrm{P}$ is at $\mathrm{O}_{1}$ point above $\mathrm{A}$ and $\mathrm{B}$, the strength of the combined electric field (hereinafter referred to as self-generated electric field) of $\mathrm{A}$ is the biggest. But in the situation, the electric field on the $\mathrm{P}$ point between $\mathrm{R}$ and $\mathrm{L}$ is unevenly affected. To reduce the impact and enhance the stability of test (shown in the experiment), $\mathrm{P}$ is arranged $2 \mathrm{~mm}$ above $\mathrm{O}_{1}$ in the experiment (namely $\mathrm{D}=2 \times 10^{-3} \mathrm{~m}$ ).

To eliminate the impact of the induced electric field, $\mathrm{P}$ should be positioned between $\mathrm{A}$ and $\mathrm{B}$. However, it is difficult to adjust $\mathrm{P}$ rightly between $\mathrm{A}$ and $\mathrm{B}$ in the experiment. It is assumed that $\mathrm{P}$ is deviated from the middle position $\Delta$ (as specified in the figure), namely closer to A. In the case, the strength of the induced electric field of A will be bigger than that of $\mathrm{B}$ on the $\mathrm{P}$ point along the horizontal direction. We can suppose their difference is $\Delta E_{1}$. When $\mathrm{P}$ is provided with positive charge, $\mathrm{A}$ and $\mathrm{B}$ will produce negative electric field, and $\Delta E_{1}$ will be directed at $\mathrm{A}$. If $\mathrm{A}$ has the self-generated electric field, its strength on the $\mathrm{P}$ point along the horizontal direction is $\varepsilon$. If the self-generated electric field is negative, $\varepsilon$ and $\Delta E_{1}$ are of the same direction, and the combined field strength on the P point along the horizontal direction will be $\varepsilon+\Delta E_{1}$. P with positive charge will be deviated to A. In the case, we impose voltage $\mathrm{U}$ on $\mathrm{R}$ and $\mathrm{L}$ through power source so as to make $\mathrm{R}$ provided with positive charge and $\mathrm{L}$ provided with negative charge. The field strength direction generated between $\mathrm{R}$ and $\mathrm{L}$ on the $\mathrm{P}$ point is opposite to that of $\varepsilon+\Delta E_{1}$. Adjust $\mathrm{U}$ to $\mathrm{U}_{1}$, and make $\mathrm{U}_{1} / \mathrm{H}$ (H is the distance between $\mathrm{R}$ and $\mathrm{L}$ ) equal to $\varepsilon+\Delta E_{1}$ numerically. At the time, the total combined field strength on the $\mathrm{P}$ point along the horizontal direction is zero, and $\mathrm{P}$ stops deviating to $\mathrm{A}$ (if $\mathrm{U}>\mathrm{U}_{1}, \mathrm{P}$ will be deviated to $\mathrm{B}$ ). And then, $\mathrm{P}$ is provided with negative charge, and both $\mathrm{A}$ and $\mathrm{B}$ produce positive electric fields. The combined field strength $\Delta E_{2}$ on the $\mathrm{P}$ point along the horizontal direction is opposite to $\Delta E_{1}$, and also the self-generated electric field $\mathcal{E}$. We suppose $\Delta E_{2}=-\Delta E_{1}$, the total combined field strength on the P point along the horizontal direction is $\varepsilon+\Delta E_{2}=\varepsilon-\Delta E_{1}$. When $\mathcal{E}>$ $\Delta E_{1}$ (adjusted to $|\Delta E|<|\varepsilon|$ in actual experiment), $\varepsilon-\Delta E_{1}$ and $\varepsilon+\Delta E_{1}$ are of the same direction, but their values are small. Since $\mathrm{P}$ is provided with negative charge, it will be deviated to the direction opposite to that when it is provided with negative charge, namely deviating leftward (to $B$ ). When we adjust $\mathrm{U}$ to $\mathrm{U}_{2}$, making $\mathrm{U}_{2} / \mathrm{H}=\varepsilon-\Delta E_{1}$, P stops deviating leftward. We can know from $\mathrm{U}_{1} / \mathrm{H}=\varepsilon+\Delta E_{1}$ and $\mathrm{U}_{2} / \mathrm{H}=\varepsilon-\Delta E_{1},\left(\mathrm{U}_{1+}\right.$ $\left.\mathrm{U}_{2}\right) / \mathrm{H}=2 \mathcal{E}$. Hence, we determine the field strength $\mathcal{E}$ of the self-generated electric field on the $\mathrm{P}_{1}$ point along the horizontal direction through the summation of $\mathrm{U}_{1}$ and $\mathrm{U}_{2}$.

If the self-generated electric field is positive, what is different from the above experiment is that $\mathrm{P}$ will be deviated leftward (B). When $U_{1}$ and $U_{2}$ are tested, $R$ is provided with negative charge, and $L$ provided with positive charge. But we still have $\varepsilon=\left(\mathrm{U}_{1}+\mathrm{U}_{2}\right) / 2 \mathrm{H}$.

\subsection{Experiment result}

\subsubsection{Experiment 1}

A is placed transversely with $\mathrm{N}$ at left and $\mathrm{S}$ at right (or $\mathrm{S}$ at left and $\mathrm{N}$ at right) to make the distance between $\mathrm{A}$ and $\mathrm{B}$ equal to $10 \mathrm{~mm}(\mathrm{~h}=5 \mathrm{~mm})$. This is the experiment for calculating $\varepsilon_{1}^{v a}$ in 5.1.3. The beaker with positive charge after the friction with plastic cloth (the effect is better than that of silk cloth) is moved to D slowly from the position directly above $\mathrm{D}$. At the time, $\mathrm{D}$ produces negative charge, and $\mathrm{P}$ produces positive charge. When the beaker is close to D, P obtains the enough positive charge, and is deviated to A. And then, R and L are imposed with a voltage $\mathrm{U}$ to make $\mathrm{R}$ with positive charge and $\mathrm{L}$ negative charge (according to the experiment principle, the $\mathrm{U}$ should be a negative value). When $\mathrm{U}$ is small, $\mathrm{P}$ is still deviated to $\mathrm{A}$. We gradually increase $\mathrm{U}$ and repeat the above experiment, and when $\mathrm{U}=\mathrm{U}_{1}=-49 \mathrm{~V}$ (average), $\mathrm{P}$ stops deviating to A and starts to deviate to $B$. In the light of the above experiment principle, $\mathrm{U}_{1} / \mathrm{H}=-250 \mathrm{~V} / \mathrm{m}$ ( $\mathrm{H}$ is actually measured $196 \mathrm{~mm}$ ).

And then when we move a plastic cup with negative charge after the friction with paper to D slowly from the position directly above $\mathrm{D}, \mathrm{P}$ produces negative charge. At the time, opposite to the above experiment, $\mathrm{P}$ is 
deviated to B. We repeat the above procedures, and when $\mathrm{U}=\mathrm{U}_{2}=-46 \mathrm{~V}$, P stops deviating to $\mathrm{B}$ and starts to deviating to A (opposite to the above experiment). We calculate $\mathrm{U}_{2} / \mathrm{H}=-235 \mathrm{~V} / \mathrm{m}$. Hence, when $\mathrm{P}$ is at the middle position of $\mathrm{A}$ and $\mathrm{B}(\mathrm{h}=5 \mathrm{~mm})$, its field strength at the horizontal direction is represented with $\mathcal{E}_{1}{ }^{0}$. according to equation (23), there is:

Equation $\varepsilon_{1}=-(250+235) / 2=-242.5 \mathrm{~V} / \mathrm{m}$

There is calculation of field strength in 5.1.2 $\varepsilon_{1}=\left(\mathrm{E}_{\mathrm{A} 1}^{-} \mathrm{E}_{\mathrm{C} 1}^{-}\right)\left(1+\left(\mathrm{E}_{\mathrm{A} 1}^{+}+\mathrm{E}_{\mathrm{Cl}}^{+}\right) /\left(\mathrm{E}_{\mathrm{A} 1}^{-}+\mathrm{E}_{\mathrm{Cl}}^{-}\right)\right)$, in which, $\mathrm{E}_{\mathrm{A} 1}^{-} \mathrm{E}_{\mathrm{Cl}}^{-}$ have relations with $\mathrm{n}=\sqrt{1-v^{2} / c^{2}}$, namely have relations with $\mathrm{V}$. Thus, different $\mathrm{V}$ shall result in different $\varepsilon_{1}$. We select a $\mathrm{v}$ to minimize the error of calculated $\varepsilon_{1}, \varepsilon_{2}$ with experimental value $\varepsilon_{1}{ }^{0} \varepsilon_{2}{ }^{0}$, there is $\mathrm{v}=3.2 \times$ $10^{3} \mathrm{~m} / \mathrm{s}$. Substitute this $\mathrm{v}$ into $\mathrm{E}_{\mathrm{A} 1}^{-} \mathrm{E}_{\mathrm{Cl}}^{+}$, it calculate out $\varepsilon_{1}=-224.3 \mathrm{v} / \mathrm{m}$. The error between $\varepsilon_{1}$ and measured value $\varepsilon^{0}{ }_{1}$ is $7.5 \%$.

\subsubsection{Experiment 2}

When the distance between $A$ and $B$ is increased to $20 \mathrm{~mm}$ (namely $\mathrm{h}=10 \mathrm{~mm}$ ), this is the experiment for calculating $\varepsilon_{2}$ in 5.1.3. According to the procedures of Experiment 1 , we measure $\mathrm{U}_{1}=-28 \mathrm{~V}$ and $\mathrm{U}_{2}=-24 \mathrm{~V}$, and then calculate out:

$$
\varepsilon_{2}^{0}=\left(U_{1}+U_{2}\right) / H=-132.7 \mathrm{~V} / \mathrm{m}
$$

Calculate according to 5.1 .2 , substitute $\mathrm{v}=3.2 \times 10^{3} \mathrm{~m} / \mathrm{s}$ into equation (15), there is $\varepsilon_{2}=-142.6 \mathrm{v} / \mathrm{m}$. The error of $\varepsilon_{2}$ and $\varepsilon^{0}{ }_{2}$ is also $7.5 \%$.

\subsubsection{Experiment 3}

A is placed vertically with $\mathrm{N}$ at front and $\mathrm{S}$ back (or $\mathrm{S}$ at front and $\mathrm{N}$ back). The distance between $\mathrm{A}$ and $\mathrm{B}$ is adjusted to $10 \mathrm{~mm}$ (namely $\mathrm{h}=5 \mathrm{~mm}$ ). This is the calculation of $\varepsilon_{3}$ in 5.2 .

Repeat procedures of Experiment 1, and the result is opposite to that of Experiment 1. When provided with positive charge, $\mathrm{P}$ is deviated to $\mathrm{B}$. When provided with negative charge, $\mathrm{P}$ is deviated to A. Measurement of field strength is opposite to that of Experiment $1 . \mathrm{R}$ is provided with negative charge, and $\mathrm{L}$ with positive charge (according to the measurement principle, the voltage at the time should be a positive value). The experiment result is $\mathrm{U}_{1}=42 \mathrm{~V}$ and $\mathrm{U}_{2}=35 \mathrm{~V}$. We can calculate out:

$$
\varepsilon_{3}=196.4 \mathrm{~V} / \mathrm{m}
$$

According to 5.2 , under the condition of $\mathrm{v}=3.2 \times 10^{3} \mathrm{~m} / \mathrm{s}$, equation (19) calculates out $\varepsilon_{3}=514.5 \mathrm{v} / \mathrm{m}$. The discrepancy of it with measured value $\varepsilon^{0}{ }_{3}$ is $318.1 \mathrm{v} / \mathrm{m}$ and the error is $162 \%$. It is sure that the calculation result is not in line with the actual situation.

\subsubsection{Experiment 4}

We adjust the distance between $A$ and $B$ in Experiment 3 to $20 \mathrm{~mm}(\mathrm{~h}=10 \mathrm{~mm})$, which is the calculation of $\varepsilon_{4}$ in 5.2. Repeat procedures of Experiment 3 , and when $U_{1}=21 \mathrm{~V}$ and $U_{2}=17 \mathrm{~V}$, we can obtain $\varepsilon_{4}^{0}=96.9 \mathrm{~V} / \mathrm{m}$. According to the calculation method of 5.2, we substitute $\mathrm{v}=3.2 \times 10^{3} \mathrm{~m} / \mathrm{s}$ into equation (18), and obtain $\varepsilon_{4}$ $=229.9 \mathrm{~V} / \mathrm{m}$. It is known that the difference between $\varepsilon_{4}$ and $\varepsilon_{4}$ is $137 \%$. Obviously, the calculated $\varepsilon_{4}$ is not in line with the actual situation.

\subsubsection{Experiment 5, 6}

$\mathrm{A}$ is still placed as in Experiment 3. But $\mathrm{P}$ is moved downward to the central position O between $\mathrm{A}$ and $\mathrm{B}$. Adjust the distance between $A$ and $B$ to $10 \mathrm{~mm}$, and repeat procedures of Experiment 3. This is Experiment 5, equivalent to the calculation of $\varepsilon_{5}$ in 5.3. Experiment shows that no matter whether $\mathrm{P}$ is provided with positive or negative charge, $\mathrm{P}$ is always deviated to the closer $\mathrm{A}$ (or B). This indicates $|\Delta E|>\left|\varepsilon_{5}^{0}\right|$. Since we can adjust $\Delta E$ to an extremely small value ( $\mathrm{P}$ position is close to $\mathrm{O}_{1}$ ), $\varepsilon_{5}^{0}$ is necessarily small. We can suppose $\varepsilon_{5}^{0}=0$. However, in the calculation of 5.3 , if $v=3.2 \times 10^{3} \mathrm{~m} / \mathrm{s}$, we can obtain $\varepsilon_{5}^{2}=-268.3 v . \varepsilon_{5}$ is completely different from $\varepsilon_{5}^{0}$. The calculation is unscientific for sure.

In experiment 6 , we adjust the distance between $\mathrm{A}$ and $\mathrm{B}$ to $20 \mathrm{~mm}$, and repeat procedures of Experiment 5 , which is the calculation of $\varepsilon_{6}$ in 5.3. Experiment shows that the result of Experiment 6 is the same as that of Experiment 5 , namely $\varepsilon_{6}^{0}=0$. In 5.3 , when $v=3.2 \times 10^{3}, \varepsilon_{6}=-241.5 \mathrm{~V} / \mathrm{m}$. It is obvious that $\varepsilon_{6}$ is completely different from $\varepsilon_{6}^{0}$, which further proves that the calculation method in 5.3 is unpractical.

\subsubsection{Notes}

1). Experiments can not be carried out in extremely humid environment. When the humidity is too high, 
electrified body, especially positive electrified body (such as the glass cup in the experiments), can not generate electricity through friction, and also release electricity quickly.

2). In experiments, induced copper tray and tin foil strip may have residual charge, which can affect experiment result. Therefore, before the start of each experiment, we had better touch the induced copper tray to eliminate residual charge.

3). Some insulated materials are likely to carry electricity, thus affecting experiment results. Therefore, the clip bases of A and B should be made of materials unlikely to carry electricity, such as timber.

4). Air flow may, such as breathing, affect the experiments, so air flow should be avoided. In the experiments, experiment devices are surrounded by four glass plates.

\subsubsection{Explanation}

The electric field measured in the experiment is similar to electrostatic field apparently, so it is called static positive electric field or static negative electric field. As stated in the paper, the measured electric field is actually the combined electric field of the electron motion negative electric field and the corresponding proton static positive electric field.

\section{Correction of Calculation Result by Sawtooth Current Model}

\subsection{Establishment of sawtooth current model}

The above six experiments show that calculation of 5 is basically not in line with the experiment result, but the negative electrostatic field in the vicinity of side edge of surface $\mathrm{N}$ and $\mathrm{S}$ and the positive electrostatic field in the vicinity of side edge of the side surface calculated by 5 are in line with the fact. Therefore, motion electric field is shortened along the motion direction, and the electric fields with the same direction but opposite motion directions are offsetting each other. The two basic viewpoints are still positive. Therefore, the reason for calculation error must be the miss of some important factors in the calculation. The author starts to doubt that combined field strength $\varepsilon_{1}, \varepsilon_{2} \ldots \ldots$ may have something to do with the total motion electric field, namely the magnetic field. However, through the calculation of the magnetic field around the rectangle coil, the author discovers that the combined field strength does not have any regular relationship with the magnetic field.

At last, it is suspected that it is improper to take one-turn rectangle current coil as the analysis model of the cubic permanent magnet. The generation of electric field of permanent magnet is due to the rough similarity of magnetic moment orientations of each molecule in magnetic domain, and molecule current forming molecule magnetic moment is non-linear current, which is obviously different from linear current of rectangle coil. From the micro-perspective, molecules on the side surface of permanent magnet are just like cobbles on river beach. Each electron moves along the surface of cobble. To make it clear, it is supposed that four molecule currents with magnetic moment of the same direction are arranged as Figure $8 \alpha$.

It is obvious that different from electrons make horizontal linear motion, as specified in Figure $8 \alpha$, only electron motion directions at points of $\mathrm{b}, \mathrm{d}, \mathrm{f}$, and $\mathrm{h}$ are horizontal, and those at other points are not horizontal, or even vertical (a, c, e, g, i point). When analyzing equation (1) above, we point out the relationship between motion field strength E'and electrostatic field strength $\mathrm{E}$ : when $v$ is fixed, the bigger the included angle $\theta$ between $\mathrm{r}$ and $v$ is, the bigger E'is.

When analyzing the motion field strength $E_{A 3}^{-}$of surface $\mathrm{A}$ in 5.2, according to the analysis model, we regard electron motions as horizontal motion on a, b, d, f, and h points in Figure 8. Therefore, most of $\theta$ of each current surface element are smaller than the actual $\theta$. As specified in Figure $8, \theta_{1}<\theta_{2} \quad\left(\mathrm{P}_{3}\right.$ point in the figure is equivalent to $\mathrm{P}_{3}$ point in Figure 4). When $\theta$ is small, E'is small accordingly. As for 5.2, this means $E_{A 3}^{-}$is smaller than the actual value, and $\left|E_{A 3}^{+}\right|-\left|E_{A 3}^{-}\right|$bigger than the actual value. This is the rise of $\varepsilon_{3}$ in 6.2.3. Namely, $\varepsilon_{4}$ is increased as well.

As for the analysis of $\left|E_{A 5}^{-}\right|$in 5.3, $\mathrm{P}_{5}$ point is on the perpendicular bisector of surface A. According to the analysis model, current makes linear motion on surface A, equivalent to horizontal motion on the points of $b, d, f$, and $h$ in Figure 8a. When $\theta$ is calculated, the $\theta$ on $\mathrm{b}, \mathrm{d}, \mathrm{f}$, and $\mathrm{h}$ is obviously bigger than that on the points nearby. As shown in $8 \mathrm{a}, \theta_{3}>\theta_{4}, \theta$ is bigger, and $\left|E_{A S}^{-}\right|$is bigger, so $\left|\mathcal{E}_{5}\right|$ is well above the actually measured $\varepsilon_{5}$. Similarly, $\left|\varepsilon_{6}\right|$ is well above $\varepsilon_{6}$ as well.

The above analysis indicates that if we put aside the analysis model of rectangle current coil, and analyze the combined field strength of permanent magnet with the actual molecule current, we can obtain the calculation result conforming to the actual situation. However, we can not accurately know the distribution of trillions of molecule currents, and it is also very difficult to make instant calculation. So how can we find out the analysis 
model conforming to the reality through the comprehensive analysis of motion features of molecule currents? We have to consider the average self-rotation speed of electrons since magnetic inductive strength of permanent magnet does not affect the distribution law of magnetic field and combined electric field. We mainly think about the law of $\theta$ changes. Because molecule magnetic moments are unnecessarily identical, we can not embark on analyzing a molecule current to obtain the general law of $\theta$ changes. In addition, combined electric field can not be completely determined by surface molecule currents. However, no matter what the distribution law of molecule magnetic moments is, we can always, under the supposition that the combined field strength depends on motion electrons on the surface, find a $\beta$ angle indicating the average increasing speed of electron motion and a $-\beta$ angle indicating the average declining speed, thus making the $\beta$ angle identical with the change effect of the overall electron motion direction. Figure $8 \mathrm{~b}$ indicates the electron motion of the $\beta$ angle. The concrete value is natural determined by experiment result, which is just like the determination of lead wire current. The speeds of free electrons forming the current in lead wire are not completely the same, and their motion directions are different as well. The current determined by us through experiments is the average current value of all free electrons. The $\beta$ is the average value of electron motion directions of all molecule currents.

Thus, we establish a new analysis model of combined electric field of permanent magnet. Different that of analysis model of 5 , its electrons do not move along the current directly, namely linear motion, but sawtooth folded line motion shown as Figure 8b. For the convenience of explanation, we call the new analysis model sawtooth current model. For protons, the current model considers them being static. Therefore, all positive electric field strengths calculated in 5 remain unchanged.

\subsection{Correction of calculation of combined field strength in 5.1 .3 by sawtooth current model}

It is supposed in sawtooth current model that electrons move along the track of the folded line in Figure $8 \mathrm{~b}$. We have a folded line unit abc. If the length of ac is supposed to $b e d x$, and $a b+b c$ is equal to $d x$ ', we can obtain $d x^{\prime}=d x / \cos \beta$. When the current I is fixed, the distance that electrons move in the unit time remains unchanged. It takes a time of $\mathrm{dt}$ for electrons to move from a to $\mathrm{clinearly}$, and the motion along the folded line

$\mathrm{a}, \mathrm{b}, \mathrm{c}$, still costs the time of dt. If linear motion speed $v=d x / d t$, folded motion speed $v^{\prime}=d x^{\prime} / d t=v / \cos \theta$. In folded line $\rightarrow$ motion, $\rightarrow$ surface charge density of electrons $\sigma_{-}^{\prime}=-I / 2 a v^{\prime}=\sigma_{-} \cos \theta$. We suppose $f(\theta)=\left(1-v^{\prime 2} / C^{2}\right) /\left(1-\sin 2 \theta v^{\prime 2} / C^{2}\right)^{3 / 2}$. Since the inclined angle of the folded line ab is $\beta$, and that of the bc section is $-\beta$. To indicate the difference, $f(\theta)$ of ab section with a length of $d x^{\prime} / 2$ is represented with $f\left(\theta_{1}\right)$;

$f(\theta)$ of bc section with the same length is represented with $f\left(\theta_{2}\right)$ [Note: the $d x^{\prime}$ here is a scalar quantity, only indicating the length. Such a stipulation is for the purpose of simplifying the calculation.\}. Under the stipulations, $d E_{A i}^{-}$of Figure 3 in the above 5 is changed into the combination of ab section and bc section.

$$
d E_{A_{1}^{\prime}}^{-}=\frac{k \sigma_{-}^{\prime} y d y}{r^{3}} \frac{1}{2} d x^{\prime} f\left(\theta_{1}\right)+\frac{k \sigma_{-}^{\prime} y d y}{r^{3}} \frac{1}{2} d x^{\prime} f\left(\theta_{2}\right)
$$

After substituting $\sigma_{-}^{\prime}=\sigma_{-} \cos \theta, d x^{\prime}=d x / \cos \theta$ into the above equation, we can obtain:

$$
d E_{A_{1}^{\prime}}^{-}=\frac{k \sigma_{-}^{\prime} y d y d x}{r^{3}} \frac{1}{2}\left[f\left(\theta_{1}\right)+f\left(\theta_{2}\right)\right]
$$

$d x^{\prime}$ and $\sigma_{-}^{\prime}$ are removed from equation (24). The forward part of the equation $k \sigma_{-}^{\prime} y d y d x / r^{3}$ is the same as that linear motion. This is certain. No matter linear line or folded line, electric quantities of surface current element $d x d y$ or $d x^{\prime} d y$ are the same, and static electric field strengths generated by them are naturally the same.

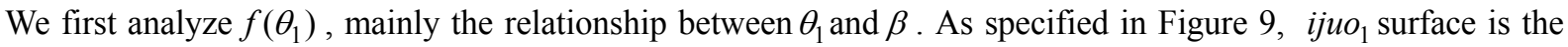
same as surface A in Figure 3 of 5.1. $\mathrm{j}$ point is the position of surface current element for the analysis of $d E_{A 1}^{-}$ in 5. In equation (24), the included angle between the extended surface $i j u^{\prime} o_{2}$ and $i j u o_{1}$ of surface current element $d y d x^{\prime} / 2$ of ab section is $\beta$. In the figure, $\mathrm{Po}_{2}$ is indicated with D'; D'is vertical with $i j u^{\prime} \mathrm{O}_{2}$ surface, so be $\triangle \mathrm{Po}_{2} u^{\prime}$. So we know $\angle p u^{\prime} j$ is a right angle; $j u^{\prime}$ is the direction of folded line electron motion speed $v^{\prime}$. So we know $\theta_{1}$ shown in the figure is the angle calculated by us. $\sin \theta_{1}=l^{\prime} / r,\left(l^{\prime}=p u^{\prime}\right)$. Four points of I, $o_{1}$, $o_{2}$, and $\mathrm{p}$ are on the same plane. If we suppose ip=S, $S=\sqrt{x^{2}+D^{2}}$, $D^{\prime}=S \sin (\alpha-\beta)=S(\sin \alpha \cos \beta-\cos \alpha \sin \beta)$. After substituting $\sin \alpha=D / S \quad \cos \alpha=x / S$ into $D^{\prime}$, we can obtain: $D^{\prime}=D \cos \beta-X \sin \beta$.

From the right angle $\Delta p o_{2} u^{\prime}$, we can know:

$$
l^{\prime}=\sqrt{y^{2}+D^{\prime 2}}=\sqrt{y^{2}+(D \cos \beta-x \sin \beta)^{2}}
$$


After substituting it into $\sin \theta_{1}$, we can obtain:

$$
\sin \theta_{1}=\frac{1}{r} \sqrt{y^{2}+(D \cos \beta-x \sin \beta)^{2}}
$$

We can analyze $f\left(\theta_{2}\right)$ with the same method. Angle $\beta$ is changed into $-\beta$, we can easily obtain:

$$
\sin \theta_{2}=\frac{1}{r} \sqrt{y^{2}+(D \cos \beta+x \sin \beta)^{2}}
$$

Substitute $\sin \theta_{1}$ and $\sin \theta_{2}$ into equation (24), we can obtain after integration:

$$
E_{A_{1}^{\prime}}^{-}=\frac{k \sigma_{-}^{\prime}}{A}\left\{\ln \frac{y^{2}+J^{2} / B^{2}}{\left(M_{1}+\sqrt{y^{2}+\frac{J^{2}}{B^{2}}+M_{1}^{2}}\right)\left(M_{2}+\sqrt{y^{2}+\frac{J^{2}}{B^{2}}+M_{1}^{2}}\right)}\right\}_{y 1}^{y 2}
$$

In the equation: $A=1-\sin ^{2} \beta v^{\prime 2} / c^{2}$

$$
\begin{gathered}
B=\frac{1-v^{\prime 2} / c^{2}}{A^{2}} \quad G=\frac{\sin 2 \beta v^{\prime 2} / c^{2}}{2 A^{2}} \\
M_{1}=\frac{a+D G}{B} \quad M_{2}=\frac{a-D G}{B} \quad J^{2}=\frac{D^{2}\left(1-\cos ^{2} \beta v^{\prime 2} / c^{2}\right)}{A^{2}}-D^{2} G^{2}
\end{gathered}
$$

$\alpha=10^{-2} \mathrm{~m}$ (half of the side length of permanent magnet); $\mathrm{D}=2 \times 10^{-3} \mathrm{~m}, \mathrm{y}_{1}=\mathrm{h} ; \mathrm{y}_{2}=\mathrm{h}+2 \alpha$. When $E_{A 1}^{-}$is calculated, $\mathrm{h}=5 \times 10^{-3} \mathrm{~m}$; When $E_{A 2^{\prime}}^{-}$is calculated, $\mathrm{h}=10^{-2} \mathrm{~m}$. ( $\beta$ value is determined according to the experiment result, see 7.5).

$E_{C 1^{\prime}}^{-}, E_{C 2^{\prime}}^{-}$, and $E_{A 1^{\prime}}^{-}$share the same equation (27). We just need to change $\mathrm{D}$ in the equation into D+2a. $\mathrm{y}_{1}$ and $\mathrm{y}_{2}$ of $E_{C 1^{\prime}}^{-}$are the same as those of $E_{A 1^{\prime}}^{-} ; \mathrm{y}_{1}$ and $\mathrm{y}_{2}$ of $E_{C 2^{\prime}}^{-}$are the same as those of $E_{A 2^{\prime}}^{-}$.

After $E_{A 1^{\prime}}^{-}, E_{A 2^{\prime}}^{-}, E_{C 1^{\prime}}^{-}$, and $E_{C 2^{\prime}}^{-}$are determined through calculation, we can calculate the combined field strength $\varepsilon_{1^{\prime}}$ and $\varepsilon_{2^{\prime}}$ corresponding with Experiment 1 and 2 according to equation (15) ( $v^{\prime}$ in $\varepsilon_{1^{\prime}}$ and $\varepsilon_{2^{\prime}}$ is determined according to the experiment result).

\subsection{Correction of calculation of combined field strength in 5.2 by sawtooth current model}

Referring to the analysis of 7.2, when calculating the combined field strength of surface A and C in 5.2 according to sawtooth current model, we can obtain:

$$
d E_{A 3^{\prime}}^{-}=\frac{k \sigma_{-} y d y d x}{r^{3}} \frac{1}{2}\left[f\left(\theta_{3}\right)+f\left(\theta_{4}\right)\right]
$$

Different from that in 7.2, the general direction of $v^{\prime}$ in 7.3 is not vertical with y axis but in parallel with y axis. Referring to the calculation of $\sin \theta_{1}$ and $\sin \theta_{2}$, we can obtain (calculation is omitted):

$$
\begin{aligned}
& \sin \theta_{3}=\frac{1}{r} \sqrt{x^{2}+(D \cos \beta-y \sin \beta)^{2}} \\
& \sin \theta_{4}=\frac{1}{r} \sqrt{x^{2}+(D \cos \beta+y \sin \beta)^{2}}
\end{aligned}
$$

[if $\mathrm{x}$ axis in Figure 9 is regarded as y axis, and y regarded as $\mathrm{x}$ axis, we can think equations (29) and (30) are similar to (25) and (26)].

After substituting (29) and (30) into (28), we can obtain through integration: 


$$
\begin{aligned}
& E_{A_{3}^{\prime}}^{-}=\frac{k \sigma_{-}^{\prime} n^{\prime}}{A^{2}}\left\{\ln \frac{\sqrt{y^{\prime 2}+J^{2}}}{\frac{a n^{\prime}}{A}+\sqrt{y^{\prime 2}+J^{2}+\frac{a^{2} n^{\prime 2}}{A^{2}}}}\right\}_{y_{1}^{\prime}}^{y_{2}^{\prime}} \\
& +\frac{k \sigma_{-}^{\prime} n^{\prime}}{A^{2}}\left\{\ln \frac{\sqrt{y^{\prime \prime 2}+J^{2}}}{\frac{a n^{\prime}}{A}+\sqrt{y^{\prime \prime 2}+J^{2}+\frac{a^{2} n^{\prime 2}}{A^{2}}}}\right\}_{y_{1}^{\prime \prime}}^{y_{2}^{\prime}}
\end{aligned}
$$

In the equation, $n^{\prime}=\sqrt{1-v^{\prime 2} / C^{2}} ; y^{\prime}=y+D G$;

$y^{\prime \prime}=y-D G, \mathrm{~A}, \mathrm{~J}$, and $\mathrm{G}$ are the same as $\mathrm{A}, \mathrm{J}$, and $\mathrm{G}$ in equation (27).

$\mathrm{y}_{1}=\mathrm{h}$ and $\mathrm{y}_{2}=\mathrm{h}+2 \mathrm{a}$; when $E_{A 3^{\prime}}^{-}$is calculated, $\mathrm{h}=5 \times 10^{-3} \mathrm{~m}$;

When $E_{A 4^{\prime}}^{-}$is calculated, $\mathrm{h}=10^{-2} \mathrm{~m} . \quad E_{C 3^{\prime}}^{-}$and $E_{C 4^{\prime}}^{-}$are calculated with equation (31) as well. The only difference is that $\mathrm{D}$ in the equation has to be changed into $\mathrm{D}+2 \mathrm{a}$.

Similarly, the combined field strength of surface B and D in 5.2 should be:

$$
d E_{B 3^{\prime}}^{-}=\frac{k \sigma_{-} h d z d x}{r^{3}} \frac{1}{2}\left[f\left(\theta_{5}\right)+f\left(\theta_{6}\right)\right]
$$

After the comparison with surface A, we can know h of surface B is equivalent to D of surface A, $\mathrm{z}$ of surface B equivalent to $\mathrm{y}$ of surface $\mathrm{A}$. Based on the relationship, we can know from the relational expression of $\sin \theta_{3}$ and $\sin \theta_{4}$ :

$$
\begin{aligned}
\sin \theta_{5} & =\frac{1}{r_{1}} \sqrt{x^{2}+(h \cos \beta-z \sin \beta)^{2}} \\
\sin \theta_{6} & =\frac{1}{r} \sqrt{x^{2}+(h \cos \beta+z \sin \beta)^{2}}
\end{aligned}
$$

After substituting $\sin \theta_{5}$ and $\sin \theta_{6}$ into equation (32), we can obtain through integration and simplification:

$$
\left\lceil E_{B 3^{\prime}}^{-}=k \sigma_{-}^{\prime} \sin ^{-1} \sqrt{\frac{(a+H)^{2}}{(a+H)^{2}+S^{2}}} \sin \operatorname{tg}^{-1} \frac{n^{\prime} z}{S A}+\sin ^{-1} \sqrt{\frac{(a-H)^{2}}{(a-H)^{2}+S^{2}}} \sin \operatorname{tg}^{-1} \frac{n^{\prime} z}{S A}\right]_{\mathrm{D}}^{D+2 a}
$$

In the equation: $H=\frac{h \sin 2 \beta v^{\prime 2} / C^{2}}{2 A^{2}} \quad n^{\prime}=\sqrt{1-v^{\prime 2} / C^{2}} \quad D=\frac{h^{2}}{A^{2}}\left(1-\cos ^{2} \beta v^{\prime 2} / C^{2}\right)-H^{2}$

When $E_{B 3^{\prime}}^{-}$is calculated, $\mathrm{h}=5 \times 10^{-3} \mathrm{~m}$; when $E_{B 4^{\prime}}^{-}$is calculated, $\mathrm{h}=10^{-2} \mathrm{~m} ; E_{D 3^{\prime}}^{-}$and $E_{D 4^{\prime}}^{-}$are calculated with equation (35) as well, and only $\mathrm{h}$ in the equation is changed into $\mathrm{h}+2 \mathrm{a}$.

With $E_{A 3^{\prime}}^{-}, E_{A 4^{\prime}}^{-}, E_{C 3^{\prime}}^{-}, E_{C 4^{\prime}}^{-}$and $E_{A 3}^{+}, E_{A 4}^{+}, E_{C 3}^{+}, E_{C 4}^{+}$, we can apply equation (15) to calculate out $\varepsilon_{A C 3}^{\prime}$ and $\varepsilon_{A C 4}$.

With $E_{B 3^{\prime}}^{-}, E_{B 4^{\prime}}^{-}, E_{D 3^{\prime}}^{-}, E_{D 4^{\prime}}^{-}$and $E_{B 3}^{+}, E_{B 4}^{+}, E_{D 3}^{+}, E_{D 4}^{+}$, we can apply equation (15) to calculate out $\varepsilon_{B D 3}^{\prime}$ and $\varepsilon_{B D 4}$. At last, we can calculate out $\mathcal{E}_{3^{\prime}}$ and $\mathcal{E}_{4^{\prime}}$ corresponding with Experiment 3 and 4 :

$$
\begin{aligned}
& \mathcal{E}_{3^{\prime}}=\boldsymbol{E}_{A C 3}^{\prime}+\boldsymbol{E}_{B D 3}^{\prime} \\
& \mathcal{E}_{4^{\prime}}=\boldsymbol{E}_{A C 4}^{\prime}+\boldsymbol{E}_{B D 4}^{\prime}
\end{aligned}
$$

7.4 Correction of calculation of combined field strength in 5.3 by sawtooth current model

Based on the above explanation, when calculating the combined field strength in 5.2 according to sawtooth current model, we can obtain:

$$
d E_{A 5^{\prime}}^{-}=\frac{k \sigma_{-} d z d y h}{r^{3}} \frac{1}{2}\left[f\left(\theta_{7}\right)+f\left(\theta_{\mathbf{8}}\right)\right]
$$

After the comparison with Figure 4 and 5, we can see $\theta_{7}$ and $\theta_{8}$ of $d E_{A 5^{\prime}}^{-}$are $\theta_{5}$ and $\theta_{6}$ of $d E_{B 3^{\prime}}^{-}$. So (38) and (32) are the same equation, and but only the integration limits of their combined field strength are different, so be the calculated values; After substituting the integration limit of $E_{A 5^{\prime}}^{-}$into equation (5), we can know: 


$$
\left\lceil E_{A 5^{\prime}}^{-}=k \sigma_{-}^{\prime} \sin ^{-1} \sqrt{\frac{(a+H)^{2}}{(a+H)^{2}+S^{2}}} \sin \operatorname{tg}^{-1} \frac{n^{\prime} z}{S A}+\sin ^{-1} \sqrt{\frac{(a-H)^{2}}{(a-H)^{2}+S^{2}}} \sin \operatorname{tg}^{-1} \frac{n^{\prime} z}{S A}\right]_{-a}^{a}
$$

When $E_{A 5^{\prime}}^{-}$is calculated, $\mathrm{h}=5 \times 10^{-3} \mathrm{~m}$; when $E_{A 6^{\prime}}^{-}$is calculated, $\mathrm{h}=10^{-2} \mathrm{~m} . E_{C 5^{\prime}}^{-}$and $E_{C 6^{\prime}}^{-}$are calculated with equation (39) as well, but h should be changed into h+2a. After calculating out $E_{A 5^{\prime}}^{-}, E_{A 6^{\prime}}^{-}$and $E_{C 5^{\prime}}^{-}$, and $E_{C 6^{\prime}}^{-}$, we can apply equation (15) to calculate out $\varepsilon_{5^{\prime}}$ and $\varepsilon_{6^{\prime}}$ corresponding with Experiment 5 and 6.

\subsection{Sawtooth current calculation result}

The above introduces each calculation equation of combined field strength on the basis of sawtooth current model, and in the next step, we will determine the $\beta$ and $v^{\prime}$ in the equation according to experiment result. The calculation result of the above 5 is a special case when $\beta=0$. Obviously, $\beta$ at the time is greatly different from the actual value. In the light of the normal seeking method, the author assumes different $\beta$ values, calculating out the combined field strengths corresponding with the above six experiments with equation (27), (31), (35), (39), and (18), and analyzing whether the calculated combined field strengths are consistent with the experiment results so as to determine whether the supposed $\beta$ value is suitable. The authors assumes that $\beta$ value is $45^{\circ}, 20^{\circ}$, $39^{\circ}, 30^{\circ}, 36^{\circ}, 35^{\circ}$, and $35.2^{\circ}$ separately. When $\beta=35.2^{\circ}$, the calculated result is generally consistent with the experiment result, explained as follows (calculation condition: $\beta=35.2^{\circ} ; v^{\prime}=3.2045 \times 10^{3} \mathrm{~m} / \mathrm{s}$ ); $\varepsilon_{1^{\prime}}$ $=-238.73 \mathrm{~V} / \mathrm{m}$;

$$
\begin{array}{ll}
\varepsilon_{2^{\prime}}=-134.76 \mathrm{v} / m ; & \varepsilon_{3^{\prime}}=+186.9 \mathrm{v} / m ; \\
\varepsilon_{4^{\prime}}=+106 \mathrm{v} / m ; & \varepsilon_{\mathbf{5}^{\prime}}=-0.926 \mathrm{v} / m ;
\end{array}
$$

$\varepsilon_{6^{\prime}}=-0.59 \mathrm{v} / \mathrm{m}$. The error between $\varepsilon_{1^{\prime}}, \varepsilon_{2^{\prime}}$ and the actually measured $\varepsilon_{1}, \varepsilon_{2}$ is $1.55 \%$. The errors of $\varepsilon_{3^{\prime}}$ and $\varepsilon_{4^{\prime}}$ are $4.8 \%$ and $9.4 \%$ respectively. Both $\varepsilon_{5^{\prime}}$ and $\varepsilon_{6^{\prime}}$ are smaller than $1 \mathrm{v} / \mathrm{m}$, considered to be the same as the actually measured $\varepsilon_{5}$ and $\varepsilon_{6}$ (we only find in the experiments that $\varepsilon_{5}$ and $\varepsilon_{6}$ are very small, and $\varepsilon_{5^{\prime}}$ and $\varepsilon_{6^{\prime}}$ are in line with the condition. $\varepsilon_{5}=\varepsilon_{6}=0$ is a supposition in 6.2.5. $\beta=35.25^{\circ}$ conforms to the supposition. The author thinks it is unnecessary to make a great amount of calculation for the supposition.)

From the above calculation we can find out that the error between $\varepsilon_{4^{\prime}}$ and actually measured value $\varepsilon_{4}$ is big (9.4\%). This indicates that the calculation method in the paper has to be further improved. Of course, this might result from the inaccurate test method (It is inaccurate that $\left|\Delta E_{1}\right|=\left|\Delta E_{2}\right|$ in the experiments; since the measured values of $U_{1}$ and $U_{2}$ can not be continuous, $U_{1}$ and $U_{2}$ are somewhat different from the actual values for sure). With a view to the fact that the forecast of the existence of the self-generated electric field accords with the fact, the calculation result of sawtooth current model is roughly identical with the experiment result, and we can obtain much accurate result if the calculation is based on the true distribution of molecule currents, we conclude: the electric field presents length contraction according to the proportion of $\sqrt{1-v^{2} / C^{2}}$ along the motion direction; the superposition principle of electrostatic field is also applicable to non-electrified body; the motion states of two electric fields having same direction and opposite direction of motion cancel out each other. The three basic viewpoints are tenable.

\section{References}

Compilation Group of "Physics" in Fudan University. Physics. (1985). High Education Press.

Teaching and Research Group for "Dynamics on Electricity" in Tsinghua University. (1964). Dynamics on Electricity. High Education Press.

Wu Shouhuang. (1987). Fundamentals on Theory of Relativity. Press of Science and Technology of Shaanxi Province. 


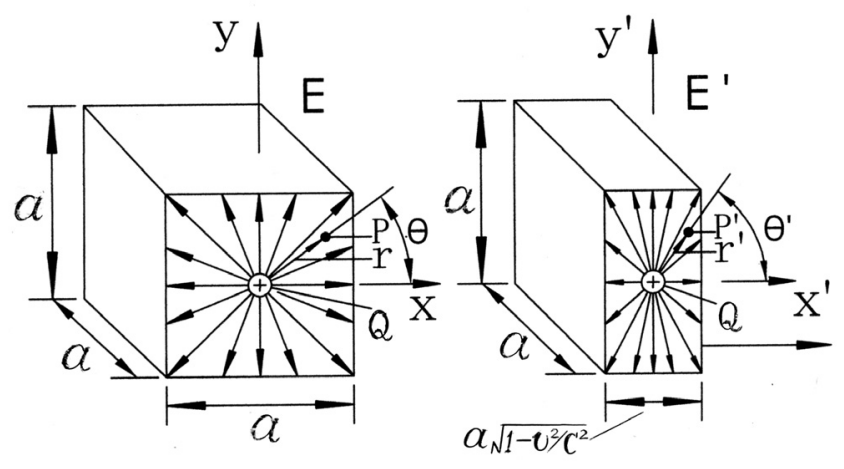

A

B

Figure 1. Length contraction of electric field in the direction of movement

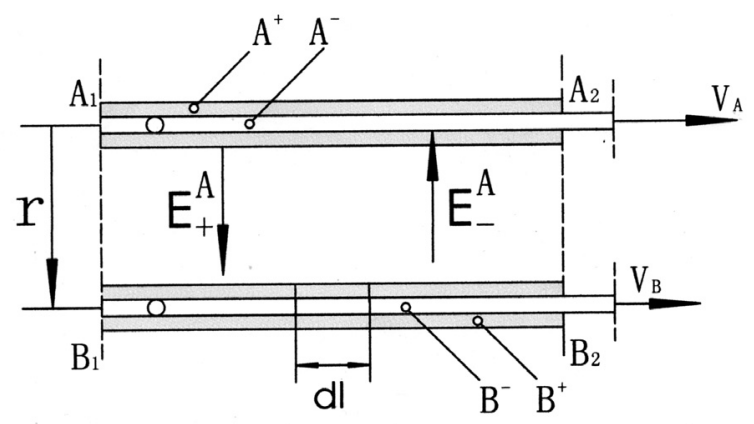

Figure 2. Interaction force of parallel current-carrying wires

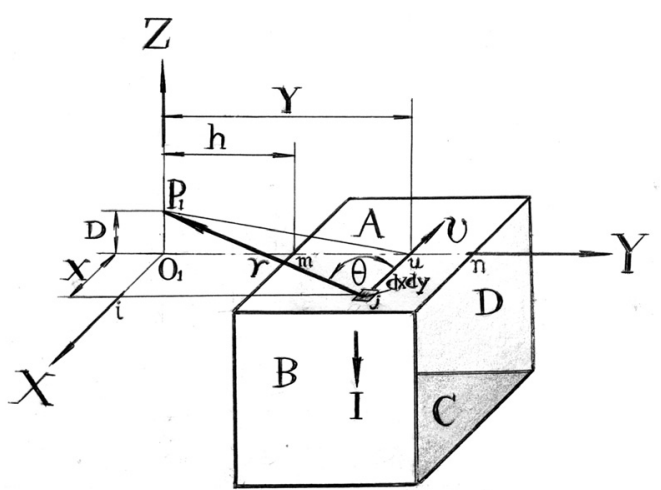

Figure 3. Analysis of combined field strength along the N.S direction outside coil 


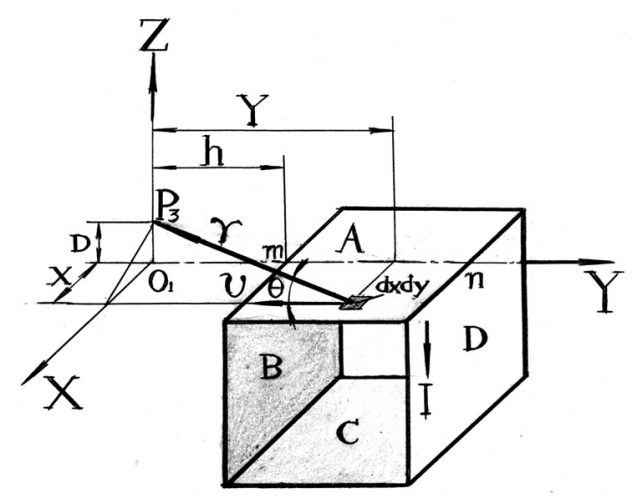

Figure 4. Analysis of combined field strength vertical with the $\mathrm{N} \bullet \mathrm{S}$ direction outside the coil

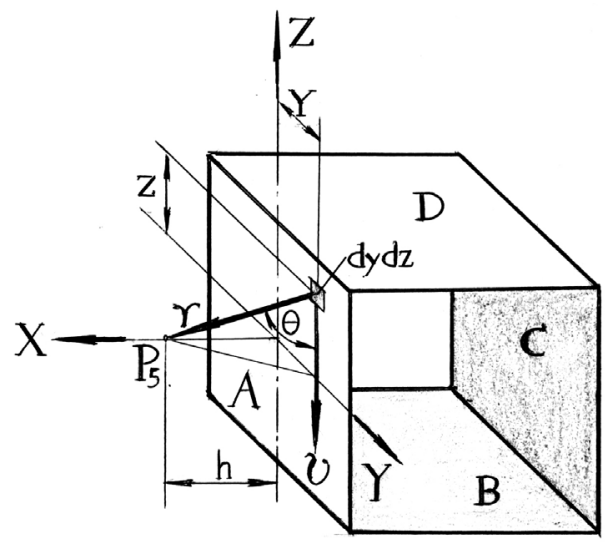

Figure 5. Analysis of combined field strength vertical with the central direction of the side of the coil

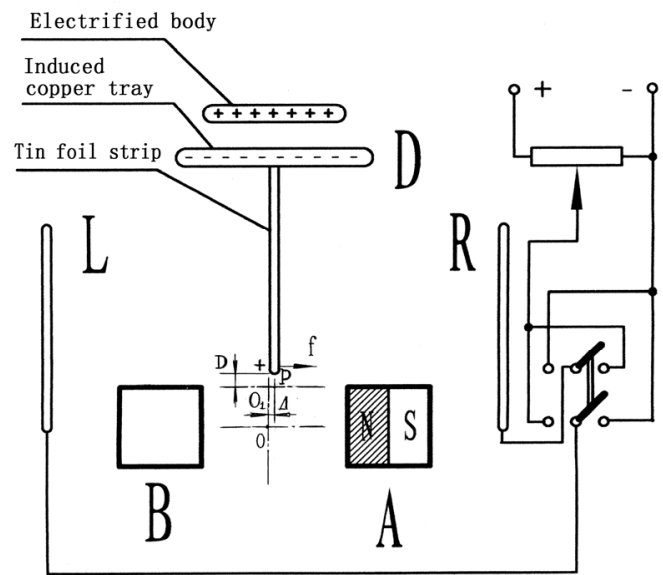

Figure 6. 


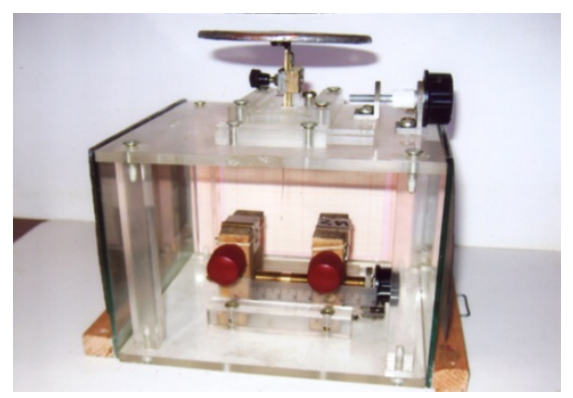

Figure 7. Photo of experiment equipment

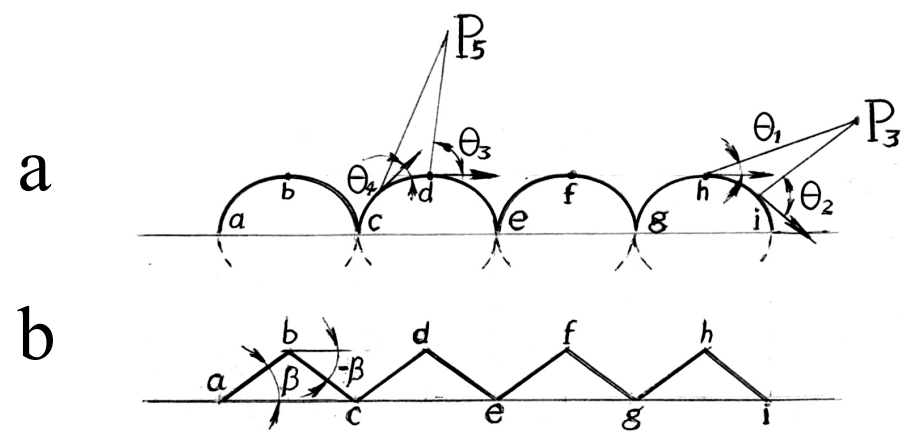

Figure 8. Replacement of true current by sawtooth current

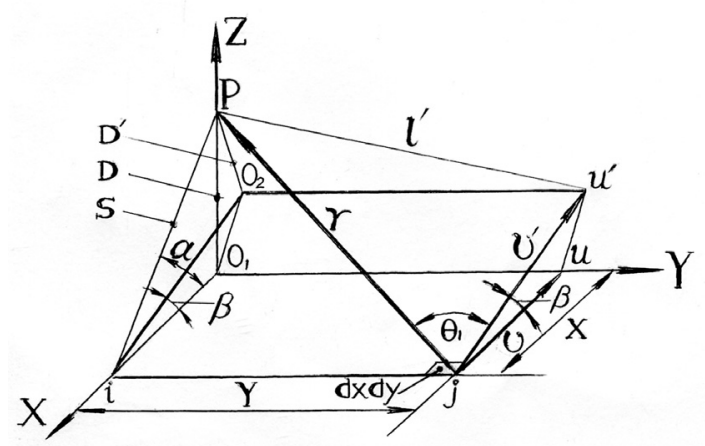

Figure 9. Figure for the analysis of relationship between $\sin \theta$ and $\beta$ 